Review

\title{
Linking gut microbiota to cardiovascular disease and hypertension: Lessons from chronic kidney disease
}

\author{
B. Meijers ${ }^{\mathrm{a}, \mathrm{b}}, \mathrm{F}$. Jouret ${ }^{\mathrm{c}, \mathrm{d}}, \mathrm{P}$. Evenepoel Eve, $^{\mathrm{a}, \mathrm{*}}$ \\ ${ }^{a}$ Nephrology Unit, University Hospitals Leuven, Herestraat 49, B-3000 Leuven, Belgium \\ b Department of Immunology and Microbiology, KU Leuven, Herestraat 49, B-3000 Leuven, Belgium \\ ${ }^{\mathrm{c}}$ Division of Nephrology, Department of Internal Medicine, University of Liège Hospital (ULg CHU), Liège, Belgium \\ ${ }^{\mathrm{d}}$ Groupe Interdisciplinaire de Génoprotéomique Appliquée (GIGA), Cardiovascular Sciences, University of Liège, Liège, Belgium
}

\section{A R T I C L E I N F O}

\section{Keywords:}

Dysbiosis

Hypertension

Fermentation

Cardiovascular disease

Microbiota

Inflammation

\begin{abstract}
A B S T R A C T
Bidirectional interactions exist between the kidneys and the gut. These interactions are commonly referred to as the gut-kidney axis. Chronic kidney disease (CKD) leads to disturbances of the gut ecosystem. Key features include the increase of protein fermentation at the expense of carbohydrate fermentation and a disrupted epithelial barrier. A disturbed gut ecosystem may contribute to the high burden of cardiovascular disease in patients with CKD. The present review discusses the impact of CKD on the gut microenvironment and provides an update as to how gut dysbiosis and a leaky gut may be linked to accelerated cardiovascular disease and hypertension.
\end{abstract}

\section{Gut microbiota in CKD}

The human intestinal tract, and especially the large intestine, is colonized by trillions of microorganisms. These microorganisms encode at least 150 -fold more genes than the human genome. The combined genetic potential of the endogenous flora is referred to as the 'microbiome' [1]. The composition of the microbial communities shows huge inter-individual variation. This variation is driven by host factors as well as environmental influences [1]. Although the microbiota is exposed to a continuously changing environment, its composition and function in an individual are stable against perturbations [2]. The microbiome provides the host with enhanced metabolic capabilities, protection against pathogens, education of the immune system, and modulation of gastrointestinal development[3].

Several chronic disabilities, including obesity and inflammatory bowel disease $[4,5]$, have been documented to go along with profound changes of the intestinal microbiota composition. The impact of chronic kidney disease (CKD) on the gut microbiome has only been explored in recent years. Vaziri et al. studied the microbial composition in stool samples and observed marked differences in the abundance of 190 bacterial operational taxonomic units (OTUs) between the end stage renal disease (ESRD) and control groups [6]. These findings were recently confirmed in a cohort of Chinese ESRD patients. The absolute quantification of total bacteria was significantly reduced, while diversity at the phylum level was maintained [7].

It should be emphasized that the renal phenotype is much broader than the loss of kidney function, as almost all patients in ESRD are on a multidrug regimen, are exposed to the effects of extracorporeal treatments and are prescribed dietary restrictions. Testing the isolated effect of the loss of kidney function on microbiome composition is only possible in experimental models. In several studies exploring the effects of surgically induced CKD in rats, total richness was reduced in CKD and the microbial community was differentially structured $[6,8]$. Andersen et al. performed a similar analysis in collagen type IV $\alpha 3$-deficient mice with Alport syndrome-related progressive CKD. Noteworthy and opposite to findings in the Chinese patient cohort, they observed a higher amount of colony forming units per gram of feces. Again, they observed differentially structured communities, with changes in the class of Bacteroidales, Burholderiales (family of Alcaligenaceae), Enterobacteriales, and Verrucomicrobiales [9].

In aggregate, these studies show that ESRD promotes alterations in the composition of the gut microbiota. This is commonly referred to as dysbiosis. At present, uncertainties remain as to the underlying pathophysiology.

\section{Gut microbial metabolism in CKD}

The gut microbial metabolism provides a significant and unique contribution towards the human metabolome [3]. The gut microbial metabolism is complex and only partly dependent on gut microbial composition. Indeed, despite variation in community structure, the metagenomic carriage of metabolic pathways has been shown to be

\footnotetext{
* Corresponding author at: Dienst nefrologie, Universitair Ziekenhuis Gasthuisberg, Herestraat 49, B-3000, Leuven, Belgium.

E-mail address: Pieter.Evenepoel@uzleuven.be (P. Evenepoel).
} 
stable, at least among healthy individuals [10].

It is generally accepted that carbohydrate and nitrogen availability is the most important determinant of colonic microbial metabolism. Dietary fiber composes the main carbohydrate supply in the colon. Other sources include oligosaccharides and a variety of sugars and nonabsorbable sugar alcohols [11,12]. Nitrogen is provided to the large intestine from exogenous dietary proteins that escaped digestion in the small intestine, endogenous proteins and from blood urea that has diffused into the intestinal lumen [13]. The colonic handling of $\alpha$ amino nitrogen (amino acids and intermediates) largely depends on the amount of energy available for bacterial growth and cell division. This energy mainly results from carbohydrate fermentation. Abundancy of carbohydrates promotes expansion of the bacterial biomass, requiring incorporation of $\alpha$-amino nitrogen. In case of carbohydrate deprivation, $\alpha$-amino nitrogen is predominantly fermented, generating potentially toxic end-products such as phenols and indoles.

The ratio of available carbohydrate to nitrogen progressively declines from proximal to distal colon, affecting regional bacterial composition and metabolism [14,15]. Slowing down colonic transit times may induce upstream expansion of proteolytic species, as a larger part of the colon will become deprived of carbohydrates, and this may result in increased generation of bacterial toxins. In a landmark study by Cummings et al., the urinary excretion rate of phenols was found to be related mainly to the colonic transit time as well as to the dietary protein intake [16].

Nutrient intake differs substantially between healthy individuals and patients with CKD, especially those with ESRD. Dietary recommendations in CKD may include restricted intake of potassium, sodium, phosphate and/or (animal) protein restriction. Such diets are low in fruit and vegetables and thus low in fermentable carbohydrates [17]. Also, the colonic transit time is prolonged in many patients with CKD. Constipation has been reported in $>50 \%$ of patients on HD and in up to $29 \%$ of patients treated with continuous ambulatory peritoneal dialysis (CAPD). These prevalences are substantial higher than reported in healthy persons $(10-20 \%)[18,19]$. As a consequence of the changes in diet and colonic transit time, microbial metabolism moves towards a predominantly proteolytic fermentation pattern. This metabolic shift aligns with the data from a metagenomics analysis on stool samples from patients with ESRD showing a significant expansion of bacterial families possessing urease, uricase, and indole and p-cresol forming enzymes, and contraction of families possessing butyrate-forming enzymes (e.g. Roseburiae, Faecalibacterium prausnitzii, Lactobacillaceae and Prevotellaceae) $[20,21]$.

The intestinal microbial metabolism is difficult to study in situ. Using a metabolomic approach, we observed major differences between patients treated with HD and healthy individuals. At least part of the observed differences could be attributed to differences in nutrient intake, as the fecal microbial metabolite profile of patients closely resembled that of household contacts following the same dietary restrictions [22]. As discussed earlier, experimental models of CKD can be used to separately study the effect of loss of kidney function on the gut microbial metabolism, irrespective from other aspects of the renal phenotype. In a 5/6th nephrectomy model in rat, significant alterations in the gut metabolites were found, including reduced abundancy of the short-chain fatty acid propanoic (also known as proprionic) acid [22]. Similar findings were noted in an adenine-induced mouse model of CKD, in which production of both propionic acid and butyric acid was reduced [23].

Mishima et al. recently studied the metabolic fingerprint in an adenine-induced mouse model of CKD, and thereby compared germfree and specific pathogen free animals with conventional animals [23]. Using this elegant double-variable experimental model these investigators were able to determine the origin of the metabolites: diet, mammalian or microbial metabolism. Several protein-fermentation metabolites including indoxyl sulphate (IndS), p-cresyl sulphate (PCS), phenyl sulphate, cholate and hippurate all were shown to originate exclusively from microbial metabolism [23].

In summary, current evidence suggests that CKD may shift microbial metabolism towards a predominantly proteolytic fermentation pattern. The clinical observation of $24 \mathrm{~h}$-urinary excretion of p-cresol paralleling renal function deterioration, supports the notion of CKD being a state of increased protein fermentation [24].

\section{Intestinal barrier in CKD}

The intestinal barrier is the master regulator, separating self from non-self, and coordinating all interactions between the gut microbiome and human physiology [25].

To prevent paracellular flux of luminal solutes, the intestinal epithelial cells are sealed together by apical junctional complexes, consisting of the tight junction, which is the most luminal component of the complex, and the subjacent adherens junction. The tight junction is selectively permeable and is able to discriminate between solutes on the basis of size and charge. While most research has focused on intestinal transcellular transport [26], recent advances in the understanding of the apical junctional complex and tight junction underpin the importance of the paracellular intestinal transport in health and in disease.

It has been known for a long time that the intestinal barrier function is impaired in CKD [27]. Only recently, the mechanisms underlying the increased intestinal permeability have been (partially) unraveled. Vaziri et al. observed intense infiltrates of lymphocytes into the lamina propria of animals with 5/6th nephrectomy induced CKD [28]. Tight junction disintegration in intestinal epithelial cells has been demonstrated by some [28], but not all investigators [9]. Whether CKD, similar to dietary changes [29] may cause alterations of the inner colonic mucus layer, thereby compromising the gut barrier function, remains to be studied. Uremic serum and plasma increased intestinal permeability in vitro, suggesting the involvement of a humoral component [30,9]. Both direct toxicity by uremic retention molecules (especially ammoniumhydroxide) and butyrate depletion may be involved. Also intestinal mucosal ischemia due to splanchnic hypoperfusion (e.g. triggered by intradialytic hypotension) and venous congestion due to heart failure [31] may be hypothesized to contribute to a disrupted gut epithelial barrier in the setting of CKD. Major mechanisms contributing to gut dysbiosis and a leaky gut in CKD are illustrated in Fig. 1.

\section{Consequences of disturbed gut ecosystem in CKD}

\subsection{Role of increased protein fermentation}

Protein fermentation results in the production of potentially toxic metabolites such as ammonia, amines, phenols and sulfides. Important representatives of these metabolites are p-cresol and indole. $p$-Cresol is a putrefaction metabolite of tyrosine, while indole is generated by fermentation of tryptophan. After absorption, the majority of p-cresol and indole is further metabolised and conjugated to form p-cresylsulphate (PCS) and indoxyl sulphate (IndS), respectively. Concentrations of PCS and IndS increase along the progression of CKD [32-34], both as a consequence of decreased elimination and increased production [35], to reach concentration in patients with ESRD are 10-50 fold higher than in healthy controls. Concentrations of PCS and IndS also rise with ageing [36].

It has been demonstrated in numerous experimental studies that at uremic concentrations, PCS and IndS may disturb several biological processes and confer direct toxicity in various cells and tissues. Multiple signaling cascades are activated and the generation of intracellular oxidative stress plays a central role (see Fig. 2). For detailed discussion, the reader is referred to recent excellent reviews dealing with this topic [37]. PCS and IndS may induce insulin resistance [38], trigger premature cellular senescence [39], upregulate DNA methyltransferase [40], activate the renin angiotensin aldosterone system [41], cause 


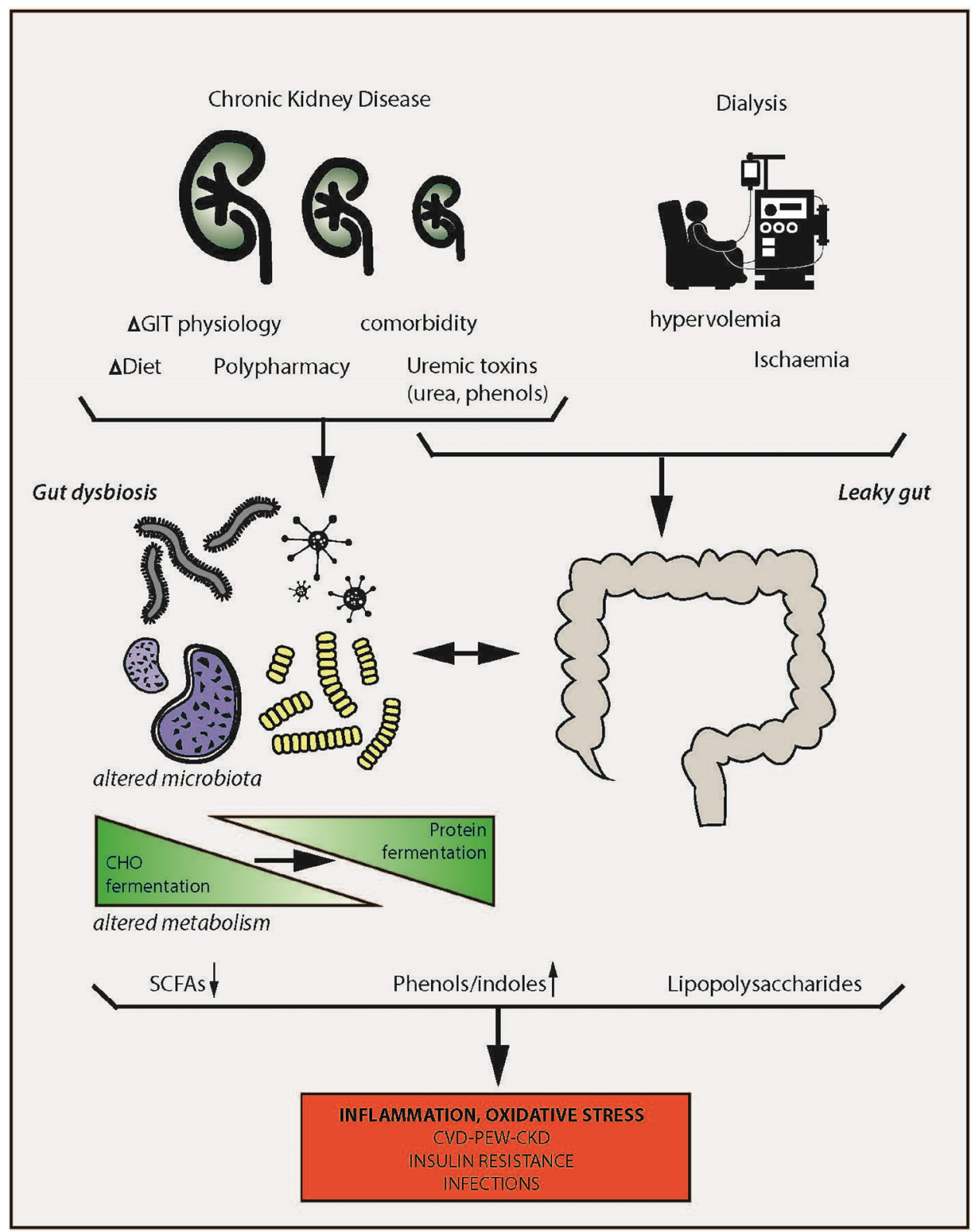

Fig. 1. Pathophysiology and consequences of gut dysbiosis and leaky gut in patients with CKD. Abbreviations: GIT: gastrointestinal tract, CHO: carbohydrate; SCFA: short chain fatty acid; CVD: cardiovascular disease; PEW: protein energy wasting; CKD: chronic kidney disease.

endothelial dysfunction [42], promote vascular calcification [43] and contribute to left ventricular hypertrophy and diastolic dysfunction. Toxicity may also be indirect as IndS and PCS, acting as sulphate sinks, may cause sulphate depletion with inherent risks. Whether uremic toxins originating from microbial metabolism trigger or sustain a systemic inflammatory reaction remains a matter of ongoing controversy. While PCS was shown to activate leucocyte free radical production [44], we failed to elicit an inflammatory response in monocytes with either PCS or IndS [45]. Moreover, clinical studies investigating the relationship between serum levels of uremic toxins originating from microbial metabolism and markers of inflammation so far yielded inconsistent findings [46].

In line with the experimental data, circulating levels of PCS and IndS have been repeatedly associated with cardiovascular morbidity (ischemic and thrombotic events, atrial fibrillation, arterial stiffness and vascular calcification) and mortality. Most evidence has been obtained in patients with CKD $[33,47,34,48]$. However also in individuals with preserved kidney function, clear associations between PCS and
IndS concentrations and cardiovascular endpoints have been reported. E.g., in a population-based study, the prevalence of hypertension increased along PCS and IndS quartiles [36]. Increased exposure to PCS and IndS may be hypothezised to be in the causal pathway between a diet rich in red meat and poor renal [49] and cardiovascular outcomes.

\subsection{Role of decreased carbohydrate fermentation}

Colonic carbohydrate fermentation results in the generation of short chain fatty acids (SCFAs), primarily acetate, propionate, and butyrate. SCFA production in the mammalian colon approximately reaches $100 \mathrm{mM}$ per day [50]. SCFA diffuse through the intestinal mucosa, circulate in the bloodstream (via the portal system; at the low-millimolar range), and act on G protein-coupled receptors (GPCR) expressed at the plasma membrane of target cells distributed widely in the mammalian organism [51,52]. Various health promoting effects have been ascribed to specific SCFAs. They may reduce the risk of developing gastrointestinal disorders, cancer, and cardiovascular disease. Hence, a 


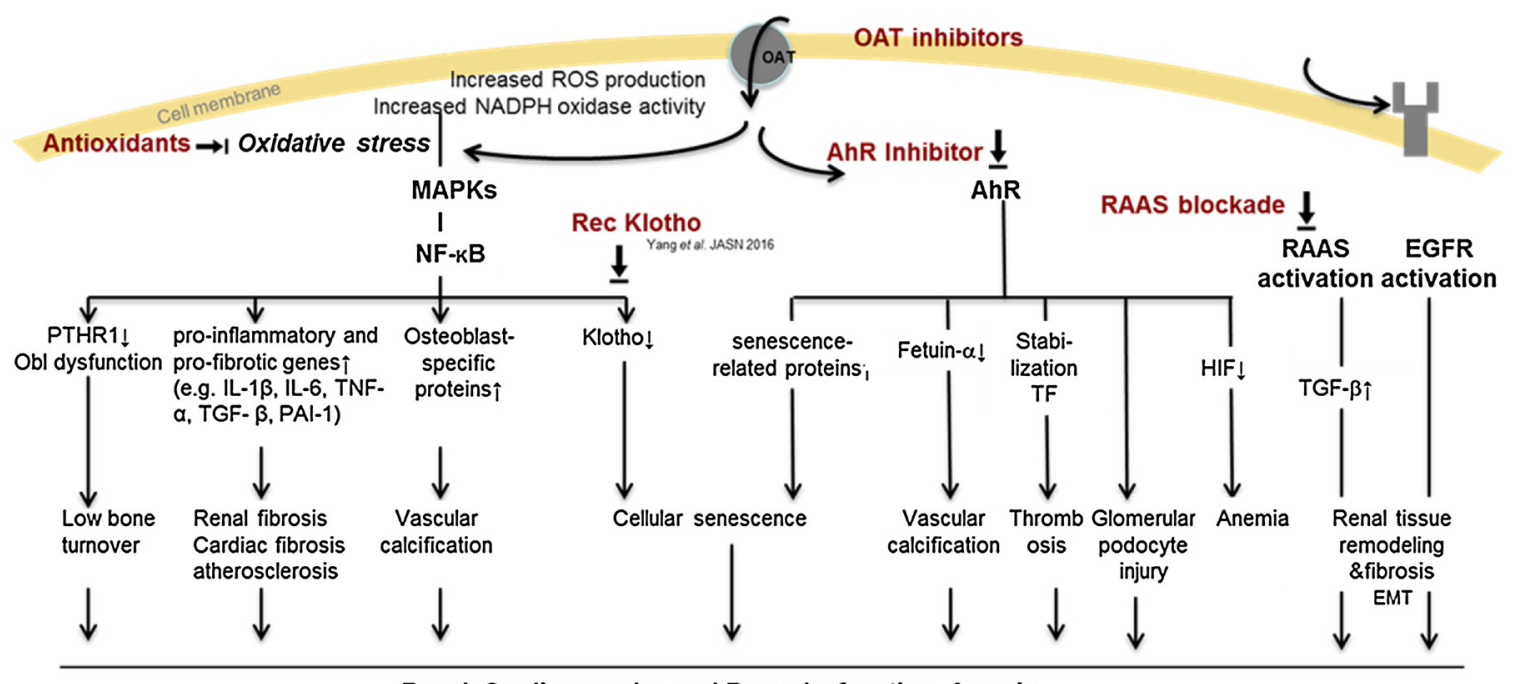

Renal, Cardiovascular, and Bone dysfunction, Anemia

Fig. 2. Signaling cascades involved in PCS and IndS mediated cellular toxicity and potential therapeutic approaches. Abbreviations: PTHR1: Parathryoid hormone receptor 1; AhR: arylhydrocarbon receptor; OAT: organic anion transporter; TF: Tissue factor; HIF: hypoxia inducible factor; Rec Klotho: recombinant Klotho; EMT: epithelial-to-mesenchymal transition; EGFR: epidermal growth factor receptor.

growing body of evidence supports that a high blood pressure (BP) is coupled to depressed carbohydrate fermentation [50]. Prescient ex vivo studies using pressure myography applied to rodent and human samples suggested that SCFA induce the vasodilation of resistance vessels in an endothelium-dependent manner [53-55]. Binding of SCFAs to the GPCR can activate a variety of intracellular signaling cascades, involving nuclear transcription, enzyme activation, and cell membrane ion transport [56]. Interestingly enough, sensory GPCR have been identified in tissues and organs not directly linked to taste or olfaction, like the kidney and the cardiovascular system [51,57-59]. Most of these GPCR are orphan receptors, i.e. with no identified endogenous ligands and functions. Still, olfactory receptor 78 (Olfr78) and Gpr41 have been recently demonstrated to act as receptors for SCFAs, with a putative role in BP control $[56,60,61]$. Of note, 2 additional SCFA GPCR are currently under investigation: Gpr109A (butyrate) and Gpr43 (acetate, propionate, and butyrate) [56].

At the mRNA level, Gpr41 is expressed in the vascular endothelium of intact vessels, where it lowers baseline BP by decreasing active vascular tone [54]. Gpr41-null mice present with hypertension of vascular origin: at baseline, plasma renin concentration is similar in Gpr41 $\mathrm{KO}$ and WT mice, and the systolic hypertension in Gpr41 KO mice is not salt-sensitive [54]. Gpr41 couples to $G_{i}$ in response to various SCFA, including propionate, formate, acetate, butyrate and isobutyrate (with an $\mathrm{EC}_{50}$ in the micromolar range) [62].

Using the LacZ reporter technique, Olfr78 has been located in resistance vessels and in the afferent arteriole in the kidney, which are physiologically involved in BP regulation [51]. Olfr78 responds to acetate and propionate, with an $\mathrm{EC}_{50}$ in the millimolar range [51]. In ex vivo experiments, SCFAs trigger renin secretion from wild-type glomeruli, but not from Olfr78-deficient glomeruli. Furthermore, mice lacking Olfr78 present with lower plasma renin and lower BP [63].

Following i.v. administration of SCFA at the millimolar range, a rapid dose-dependent drop in BP is observed in mice [63]. In Gpr41deficient animals, such a hypotensive response to SCFA infusion is lost. Conversely, SCFA-induced hypotension is aggravated in Olfr78-null mice [63]. In toto, these observations suggest that, at least in mouse, proprionate and acetate modulate the BP differentially according to the type of activated receptors: activated Gpr41 causes hypotension, whereas Olfr78 stimulation favors BP increase [64]. As a reminder, SCFA EC 50 of Gpr41 and Olfr78 significantly varies from the micro- to the millimolar range, respectively, which may partly explain why similar SCFAs target both receptors with opposite BP results. These basic science data corroborate epidemiological studies showing a significant association between the urinary levels of SCFAs (especially formate) and BP levels, and reinforce the conclusions of a meta-analysis illustrating the influence of dietary fibers on BP control $[65,66]$. Furthermore, a meta-analysis of randomized controlled trials showed that the use of probiotics (which, in turn, increase SCFA production) is associated with lower BP levels [67]. Finally, SCFA impact on BP homeostasis may also explain why the use of acetate in hemodialysis solutions causes hypotension in patients [68]. Thus, a growing body of evidence supports that BP regulation is coupled not only to host but also to microbial metabolism [69]. This conclusion is corroborated by data from a small study showing an increased Firmicutes/Bacteroidetes ratio and a decrease in acetate- and butyrate-producing bacteria in hypertensive patients [70]. Furthermore, in hypertensive rats, oral minocycline was able to restore gut microbiota, reduce the Firmicutes/ Bacteroidetes ratio, and attenuate BP levels [70].

Gut dysbiosis may thus be a relevant and so far largely neglected player in the pathogenesis of hypertension. Besides its putative role in the pathogenesis of hypertension, SCFA depletion may also contribute to micro-inflammation since SCFAs have been reported to have antiinflammatory properties by binding Gpr43 [71].

\subsection{Role of a disturbed intestinal barrier}

Dysfunction of the intestinal barrier in CKD is associated with bacterial translocation and endotoxinemia, which is related to systemic inflammation, malnutrition, cardiovascular disease, and possibly reduced survival $[28,72,73,9]$.

ESRD is associated with micro-inflammation, a chronic low-grade inflammatory state that is characterized by elevated levels of circulating pro-inflammatory cytokines, including interleukin-6 (IL-6). This condition has a multifactorial origin involving accumulation of advanced glycation end-products and oxidized protein products, acidosis, volume overload, oxidative stress, bio-incompatibility of dialyzer membrane amongst others. New evidence suggests that a dysfunctional intestinal barrier may also contribute to the micro-inflammatory state in CKD. An increased intestinal permeability results in the translocation of bacteria and bacterial cell wall components (endotoxins). This coincides with the activation of the immune system as has been demonstrated in experimental models of CKD [74]. This concept is not new, as already in 1907, Elie Metchnikoff proposed that chronic systemic inflammation may occur as a result of increased permeability and the 
escape of bacteria and their products [75]. In line with these data, recent evidence from murine studies indicates that age-associated inflammation is at least partly mediated by age-related microbiota increasing intestinal permeability. Systemic inflammation in turns contributes to microbial dysbiosis, thereby initiating a vicious circle [76].

Actions of endotoxin (lipopolysaccharide) are mediated by interaction with the Toll-like receptor 4/MD-2 complex and its co-receptor CD14 on monocytic cells, initiating an innate immune response and pro-inflammatory signaling. In clinical cohort studies, gut bacterial translocation and endotoxinemia, directly relates to markers of inflammation $[77,9]$.

A growing body of evidence indicates that endotoxinemia and micro-inflammation may be in the causal pathway between CKD and the high burden of cardiovascular disease [78,72,79-81]. This evidence, in addition, enforces the so-called inflammatory hypothesis in our understanding of the biology of atherosclerosis [82]. Inflammatory cells and signals drive the healing response to vascular injury, allowing the initiation and growth of atherosclerotic plaque. Inflammatory reactions probably increase plaque instability and setting up the substrate for the thrombotic response that causes myocardial damage or infarction, e.g. by upregulating tissue factor expression. Recent data from a RCT with canakinumab, a human monoclonal antibody against interleukin- $1 \beta$, added further credit to the inflammatory hypothesis of coronary artery disease [83].

\section{Treatment options}

A multi-targeted approach may be required to tackle the disturbed gut ecosystem in CKD. For detailed discussion, the reader is referred to several excellent reviews discussing this topic in detail [84-86].

\subsection{Restoring gut homeostasis}

Acknowledging that diet is a key regulator of microbial metabolism, one may either restrict protein intake or increase dietary fiber intake in an attempt to revert microbial metabolism towards a predominantly saccharolytic fermentation pattern. As protein restriction is unpalatable and can accentuate sarcopenia, increasing dietary fiber intake provides a safer and simpler means to restore eubiosis. Fruits and vegetables are not only rich in dietary fiber, but also in potassium, which could be problematic in patients with advanced CKD. In these patients, prebiotics (i.e a non-digestible food ingredient that promotes the growth of beneficial microorganisms in the intestines) may be an appealing alternative. Other approaches to restore microbial metabolism include treatment with laxatives (to reduce transit time), probiotics (i.e. live microorganisms that, when administered in adequate amounts, confer a health benefit on the host) or genetic manipulation of the colon microbiota (e.g. to suppress the conversion tryptophan to indole).

\subsection{Adsorption of toxic protein fermentation metabolites}

Another means to reduce the daily load of potentially toxic endproducts of protein fermentation is to administer sorbents. The oral charcoal adsorbent AST-120 (Kremezin, Kureha Ltd., Japan) reduces plasma levels of several end-products of protein fermentation including IndS and PCS. Animal studies showed beneficial effects on gut [87], renal and cardiovascular outcomes. Also pilot clinical interventions studies with AST-120 showed promising results, but renal benefits could not be confirmed in a large RCT [88]. Characteristics of the study population and compliance and statistical issues may have contributed to the negative result of the latter study. Also intake of sevelamer, a non-calcium, non-aluminum phosphate binder have been associated with lower PCS levels, but finding, overall, were inconsistent $[89,90]$.

\subsection{Targeting biological activity}

The toxicity of PCS and IndS is mediated through various signaling pathways in which the generation of intracellular oxidative stress and activation of the aryl hydrocarbon receptor (ahr) play a central role $[37,84]$. Organic anion transporters are involved in the intracellular uptake of IndS and PCS. Fig. 2 summarizes the signaling pathways and identifies candidate therapeutic targets. The rationale of inhibition of intracellular uptake and intracellular signal mediators has been demonstrated in a series of experimental studies, but clinical efficacy and safety remain to be demonstrated.

\subsection{Increasing the removal of colon-derived toxins}

Excretion of IndS, PCS and other microbiome-derived uremic retention solutes is predominantly via the kidneys, as the bile does not contain significant amounts of these solutes (unpublished data). As these solutes are tightly bound to albumin, glomerular clearance is limited and most solute is secreted by active transporter systems in the proximal tubule [91]. Active transport of organic anions is one of the genetically conserved functions of the kidney. Key transporters include the organic anion transporter 1 and 3 (OAT 1/3) as well as the organic anion transporter protein 4C1 (OATP4C1), all of which are facilitated diffusion carriers. Additional transporters include adenosinetriphosphate (ATP)-dependent pumps, i.e. P-glycoprotein (P-gp), multidrug resistance proteins 2 and $4(\mathrm{MRCP} 2 / 4)$ and breast cancer resistance protein (BRCP). Theoretically, such systems may be exploited to drive up the renal excretion of microbiome-derived uremic retention solutes. In reality, however, due to accumulation of other molecules competing for the same excretory route, elimination of the microbiome-derived molecules often is limited by saturation of the transporters.

The strong albumin binding also explains why removal by conventional hemodialysis is limited [92,93]. Alternative treatment regimens were shown to increase clearances, but their impact on circulating levels, overall, was limited if not negligible. Adsorptive techniques, though promising from an efficacy point of view, are limited by high costs and/or non-selective adsorption causing inferring a high risk of thrombotic complications $[94,95]$.

\section{Future directions and conclusions}

Over the last decade, it has become clear that the microbiome is involved in various aspects of the uremic syndrome. Host-microbiota interactions are bidirectional. The gut microbial composition is altered in patients with CKD, as is microbial metabolism with a shift towards a predominant proteolytic fermentation pattern. The intestinal barrier, shielding the human internal environment from the fascinating world of microbes, is disturbed in patients with CKD. These alterations contribute via various mechanisms to the high burden of cardiovascular disease and hypertension in patients with CKD.

The great challenges - however - lie ahead. Although it is becoming more and more evident that we need to interfere with the disturbed gut ecosystem in patients with CKD, there are no clear and effective inroads. Many questions are still in the open. It is unclear whether we should primarily interfere with gut microbial composition, gut microbial metabolic activity or both. It is not clear whether it would be possible to restore a sustained healthy microbiome. It is not even clear whether patients would benefit most from a healthy normal microbiome, or that some alterations, e.g. increased metabolic capacity to digest urea and uric acid, are necessary adaptations. The time is right to tackle these challenges, as they hold promise to find novel therapeutic targets for a challenging disease population.

\section{Conflict of interest}

None of the authors declares a conflict of interest. 


\section{References}

[1] P.J. Turnbaugh, R.E. Ley, M. Hamady, C.M. Fraser-Liggett, R. Knight, J.I. Gordon, The human microbiome project, Nature 449 (2007) 804-810.

[2] F. Sommer, J.M. Anderson, R. Bharti, J. Raes, P. Rosenstiel, The resilience of the intestinal microbiota influences health and disease, Nat. Rev. Microbiol. 15 (2017) 630-638.

[3] W.R. Wikoff, A.T. Anfora, J. Liu, P.G. Schultz, S.A. Lesley, E.C. Peters, G. Siuzdak, Metabolomics analysis reveals large effects of gut microflora on mammalian blood metabolites, Proc. Natl. Acad. Sci. U. S. A. 106 (2009) 3698-3703.

[4] V. Tremaroli, F. Backhed, Functional interactions between the gut microbiota and host metabolism, Nature 489 (2012) 242-249.

[5] M.J. Claesson, I.B. Jeffery, S. Conde, S.E. Power, E.M. O'Connor, S. Cusack, H.M. Harris, M. Coakley, B. Lakshminarayanan, O. O'Sullivan, G.F. Fitzgerald, J. Deane, M. O'Connor, N. Harnedy, K. O'Connor, D. O'Mahony, Wallace M van SD, L. Brennan, C. Stanton, J.R. Marchesi, A.P. Fitzgerald, F. Shanahan, C. Hill, R.P. Ross, P.W. O'Toole, Gut microbiota composition correlates with diet and health in the elderly, Nature 488 (2012) 178-184.

[6] N.D. Vaziri, J. Wong, M. Pahl, Y.M. Piceno, J. Yuan, T.Z. Desantis, Z. Ni, T.H. Nguyen, G.L. Andersen, Chronic kidney disease alters intestinal microbial flora, Kidney Int. (2013) 308-315.

[7] S. Jiang, S. Xie, D. Lv, P. Wang, H. He, T. Zhang, Y. Zhou, Q. Lin, H. Zhou, J. Jiang, J. Nie, F. Hou, Y. Chen, Alteration of the gut microbiota in Chinese population with chronic kidney disease, Sci. Rep. 7 (2017) 2870.

[8] M. Kikuchi, M. Ueno, Y. Itoh, W. Suda, M. Hattori, Uremic toxin-producing gut microbiota in rats with chronic kidney disease, Nephron 135 (2017) 51-60.

[9] K. Andersen, M.S. Kesper, J.A. Marschner, L. Konrad, M. Ryu, V.S. Kumar, O.P. Kulkarni, S.R. Mulay, S. Romoli, J. Demleitner, P. Schiller, A. Dietrich, S. Muller, O. Gross, H.J. Ruscheweyh, D.H. Huson, B. Stecher, H.J. Anders, Intestinal dysbiosis, barrier dysfunction, and bacterial translocation account for CKD-Related systemic inflammation, J. Am. Soc. Nephrol. 28 (2017) 76-83.

[10] Structure, function and diversity of the healthy human microbiome, Nature 486 (2012) 207-214.

[11] A. Birkett, J. Muir, J. Philips, G. Jones, K. O'Dea, Resistant starch lowers fecal concentrations of ammonia and phenols in humans, Am. J. Clin. Nutr. 63 (1996) 766-772.

[12] E. Smith, G. Macfarlane, Enumeration of human colonic bacteria producing phenolic and indolic compounds: effects of $\mathrm{pH}$, carbohydrate availability and retention time on dissimilatory aromatic amino acid metabolism, J. Appl. Bact. 81 (1996) 288-302.

[13] P. Evenepoel, B.K. Meijers, B.R. Bammens, K. Verbeke, Uremic toxins originating from colonic microbial metabolism, Kidney Int. Suppl. (2009) S12-S19.

[14] G.T. Macfarlane, J.H. Cummings, S. Macfarlane, G.R. Gibson, Influence of retention time on degradation of pancreatic enzymes by human colonic bacteria grown in a 3stage continuous culture system, J. Appl. Bacteriol. 67 (1989) 520-527.

[15] G.T. Macfarlane, S. Macfarlane, Models for intestinal fermentation: association between food components, delivery systems, bioavailability and functional interactions in the gut, Curr. Opin. Biotechnol. 18 (2007) 156-162.

[16] J. Cummings, M. Hill, E. Bone, W. Branch, D. Jenkins, The effect of meat protein and dietary fiber on colonic function and metabolism, Am. J. Clin. Nutr. 32 (1979) 2094-2101.

[17] B. Meijers, G. Glorieux, R. Poesen, S.J. Bakker, Nonextracorporeal methods for decreasing uremic solute concentration: a future way to go? Semin. Nephrol. 34 (2014) 228-243.

[18] G. Yasuda, K. Shibata, T. Takizawa, Y. Ikeda, Y. Tokita, S. Umemura, O. Tochikubo, Prevalence of constipation in continuous ambulatory peritoneal dialysis patients and comparison with hemodialysis patients, Am. J. Kidney Dis. 39 (2002) 1292-1299.

[19] M.J. Wu, C.S. Chang, C.H. Cheng, C.H. Chen, W.C. Lee, Y.H. Hsu, K.H. Shu, M.J. Tang, Colonic transit time in long-term dialysis patients, Am. J. Kidney Dis. 44 (2004) 322-327.

[20] J. Wong, Y.M. Piceno, T.Z. Desantis, M. Pahl, G.L. Andersen, N.D. Vaziri, Expansion of urease- and uricase-containing: indole- and p-cresol-forming and contraction of short-chain fatty acid-producing intestinal microbiota in ESRD, Am. J. Nephrol. 39 (2014) 230-237.

[21] S. Jiang, S. Xie, D. Lv, Y. Zhang, J. Deng, L. Zeng, Y. Chen, A reduction in the butyrate producing species Roseburia spp. and Faecalibacterium prausnitzii is associated with chronic kidney disease progression, Antonie Van Leeuwenhoek 109 (2016) 1389-1396.

[22] R. Poesen, K. Windey, E. Neven, D. Kuypers, P.V. de, P. Augustijns, P. D'Haese, P. Evenepoel, K. Verbeke, B. Meijers, The influence of CKD on colonic microbial metabolism, J. Am. Soc. Nephrol. 27 (2016) 1389-1399.

[23] E. Mishima, S. Fukuda, C. Mukawa, A. Yuri, Y. Kanemitsu, Y. Matsumoto, Y. Akiyama, N.N. Fukuda, H. Tsukamoto, K. Asaji, H. Shima, K. Kikuchi, C. Suzuki, T. Suzuki, Y. Tomioka, T. Soga, S. Ito, T. Abe, Evaluation of the impact of gut microbiota on uremic solute accumulation by a CE-TOFMS-based metabolomics approach, Kidney Int. 92 (2017) 634-645.

[24] B. Bammens, K. Verbeke, Y. Vanrenterghem, P. Evenepoel, Evidence for impaired assimilation of protein in chronic renal failure, Kidney Int. 64 (2003) 2196-2203.

[25] M.M. France, J.R. Turner, The mucosal barrier at a glance, J. Cell Sci. 130 (2017) 307-314.

[26] L.L. Wu, W.H. Peng, W.T. Kuo, C.Y. Huang, Y.H. Ni, K.S. Lu, L.C. Yu, J.R. Turner, Commensal bacterial endocytosis in epithelial cells is dependent on myosin light chain kinase-activated brush border fanning by interferon-gamma, Am. J. Pathol. 184 (2014) 2260-2274.
[27] M. Magnusson, K.E. Magnusson, T. Sundqvist, T. Denneberg, Impaired intestinal barrier function measured by differently sized polyethylene glycols in patients with chronic renal failure, Gut 32 (1991) 754-759.

[28] N.D. Vaziri, J. Yuan, A. Rahimi, Z. Ni, H. Said, V.S. Subramanian, Disintegration of colonic epithelial tight junction in uremia: a likely cause of CKD-associated inflammation, Nephrol. Dial. Transplant. 27 (2012) 2686-2693.

[29] B.O. Schroeder, G.M.H. Birchenough, M. Stahlman, L. Arike, M.E.V. Johansson, G.C. Hansson, F. Backhed, Bifidobacteria or fiber protects against diet-induced microbiota-mediated colonic mucus deterioration, Cell Host Microbe 23 (2018) 27-40.

[30] N.D. Vaziri, N. Goshtasbi, J. Yuan, S. Jellbauer, H. Moradi, M. Raffatellu, K. Kalantar-Zadeh, Uremic plasma impairs barrier function and depletes the tight junction protein constituents of intestinal epithelium, Am. J. Nephrol. 36 (2012) 438-443.

[31] A. Krack, R. Sharma, H.R. Figulla, S.D. Anker, The importance of the gastrointestinal system in the pathogenesis of heart failure, Eur. Heart J. 26 (2005) 2368-2374.

[32] B.K. Meijers, L.H. de, B. Bammens, K. Verbeke, Y. Vanrenterghem, P. Evenepoel, pCresyl sulfate and indoxyl sulfate in hemodialysis patients, Clin. J. Am. Soc. Nephrol. 4 (2009) 1932-1938.

[33] B.K. Meijers, K. Claes, B. Bammens, L.H. de, L. Viaene, K. Verbeke, D. Kuypers, Y. Vanrenterghem, P. Evenepoel, p-Cresol and cardiovascular risk in mild-to-moderate kidney disease, Clin. J. Am. Soc. Nephrol. 5 (2010) 1182-1189.

[34] F.C. Barreto, D.V. Barreto, S. Liabeuf, N. Meert, G. Glorieux, M. Temmar, G. Choukroun, R. Vanholder, Z.A. Massy, Serum indoxyl sulfate is associated with vascular disease and mortality in chronic kidney disease patients, Clin. J. Am. Soc. Nephrol. 4 (2009) 1551-1558.

[35] R. Poesen, L. Viaene, K. Verbeke, K. Claes, B. Bammens, B. Sprangers, M. Naesens, Y. Vanrenterghem, D. Kuypers, P. Evenepoel, B. Meijers, Renal clearance and intestinal generation of p-Cresyl sulfate and indoxyl sulfate in CKD, Clin. J. Am. Soc. Nephrol. 8 (2013) 1508-1514.

[36] L. Viaene, L. Thijs, Y. Jin, Y. Liu, Y. Gu, B. Meijers, K. Claes, J. Staessen, P. Evenepoel, Heritability and clinical determinants of serum indoxyl sulfate and pcresyl sulfate, candidate biomarkers of the human microbiome enterotype, PLoS One 9 (2014) e79682.

[37] R. Vanholder, E. Schepers, A. Pletinck, E.V. Nagler, G. Glorieux, The uremic toxicity of indoxyl sulfate and p-cresyl sulfate: a systematic review, J. Am. Soc. Nephrol. 25 (2014) 1897-1907.

[38] L. Koppe, N.J. Pillon, R.E. Vella, M.L. Croze, C.C. Pelletier, S. Chambert, Z. Massy, G. Glorieux, R. Vanholder, Y. Dugenet, H.A. Soula, D. Fouque, C.O. Soulage, pCresyl sulfate promotes insulin resistance associated with CKD, J. Am. Soc. Nephrol. 24 (2013) 88-99.

[39] G. Muteliefu, H. Shimizu, A. Enomoto, F. Nishijima, M. Takahashi, T. Niwa, Indoxyl sulfate promotes vascular smooth muscle cell senescence with upregulation of p53, p21, and prelamin A through oxidative stress, Am. J. Physiol. Cell Physiol. 303 (2012) C126-C134.

[40] C.Y. Sun, S.C. Chang, M.S. Wu, Suppression of Klotho expression by protein-bound uremic toxins is associated with increased DNA methyltransferase expression and DNA hypermethylation, Kidney Int. 81 (2012) 640-650.

[41] C.Y. Sun, S.C. Chang, M.S. Wu, Uremic toxins induce kidney fibrosis by activating intrarenal renin-angiotensin-aldosterone system associated epithelial-to-mesenchymal transition, PLoS One 7 (2012) e34026.

[42] B.K. Meijers, S. Van kerckhoven, k. Verbeke, W. Dehaen, Y. Vanrenterghem, M.F. Hoylaerts, P. Evenepoel, The uremic retention solute p-cresyl sulfate and markers of endothelial damage, Am. J. Kidney Dis. 54 (2009) 891-901.

[43] T. Niwa, H. Shimizu, Indoxyl sulfate induces nephrovascular senescence, J. Ren. Nutr. 22 (2012) 102-106.

[44] E. Schepers, N. Meert, G. Glorieux, J. Goeman, J. Van der Eycken, R. Vanholder, Pcresylsulphate, the main in vivo metabolite of p-cresol, activates leucocyte free radical production, Nephrol. Dial. Transplant. 22 (2007) 592-596.

[45] L. Viaene, P. Evenepoel, B. Meijers, D. Vanderschueren, L. Overbergh, C. Mathieu, Uremia suppresses immune signal-induced CYP27B1 expression in human monocytes, Am. J. Nephrol. 36 (2012) 497-508.

[46] H.J. Hsu, C.H. Yen, I.W. Wu, K.H. Hsu, C.K. Chen, C.Y. Sun, C.C. Chou, C.Y. Chen, C.J. Tsai, M.S. Wu, C.C. Lee, The association of uremic toxins and inflammation in hemodialysis patients, PLoS One 9 (2018) 2014-e102691.

[47] B.K.I. Meijers, B. Bammens, B. De Moor, K. Verbeke, Y. Vanrenterghem, P. Evenepoel, Free serum concentrations of p-cresol are independently associated with cardiovascular disease in hemodialysis, Kindey Int. 73 (2008) 1174-1180.

[48] S. Liabeuf, D.V. Barreto, F.C. Barreto, N. Meert, G. Glorieux, E. Schepers, M. Temmar, G. Choukroun, R. Vanholder, Z.A. Massy, Free p-cresylsulphate is a predictor of mortality in patients at different stages of chronic kidney disease, Nephrol. Dial. Transplant. 25 (2010) 1183-1191.

[49] Q.J. Lew, T.H. Jafar, H.W. Koh, A. Jin, K.Y. Chow, J.M. Yuan, W.P. Koh, Red meat intake and risk of ESRD, J. Am. Soc. Nephrol. 28 (2017) 304-312.

[50] M. Bugaut, Occurrence, absorption and metabolism of short chain fatty acids in the digestive tract of mammals, Comp. Biochem. Physiol. B 86 (1987) 439-472.

[51] J.L. Pluznick, D.J. Zou, X. Zhang, Q. Yan, D.J. Rodriguez-Gil, C. Eisner, E. Wells, C.A. Greer, T. Wang, S. Firestein, J. Schnermann, M.J. Caplan, Functional expression of the olfactory signaling system in the kidney, Proc. Natl. Acad. Sci. U. S. A. 106 (2009) 2059-2064.

[52] J. Gilissen, F. Jouret, B. Pirotte, J. Hanson, Insight into SUCNR1 (GPR91) structure and function, Pharmacol. Ther. 159 (2016) 56-65.

[53] F.V. Mortensen, H. Nielsen, M.J. Mulvany, I. Hessov, Short chain fatty acids dilate isolated human colonic resistance arteries, Gut 31 (1990) 1391-1394.

[54] N. Natarajan, D. Hori, S. Flavahan, J. Steppan, N.A. Flavahan, D.E. Berkowitz, 
J.L. Pluznick, Microbial short chain fatty acid metabolites lower blood pressure via endothelial G protein-coupled receptor 41, Physiol. Genomics 48 (2016) 826-834.

[55] C.W. Nutting, S. Islam, J.T. Daugirdas, Vasorelaxant effects of short chain fatty acid salts in rat caudal artery, Am. J. Physiol. 261 (1991) H561-H567.

[56] J. Peti-Peterdi, B.K. Kishore, J.L. Pluznick, Regulation of vascular and renal function by metabolite receptors, Annu. Rev. Physiol. 78 (2016) 391-414.

[57] J.H. Robben, R.A. Fenton, S.L. Vargas, H. Schweer, J. Peti-Peterdi, P.M. Deen, G. Milligan, Localization of the succinate receptor in the distal nephron and its signaling in polarized MDCK cells, Kidney Int. 76 (2009) 1258-1267.

[58] P. Rajkumar, W.H. Aisenberg, O.W. Acres, R.J. Protzko, J.L. Pluznick, Identification and characterization of novel renal sensory receptors, PLoS One 9 (2014) e111053.

[59] X. Liu, F. Gu, L. Jiang, F. Chen, F. Li, Expression of bitter taste receptor Tas2r105 in mouse kidney, Biochem. Biophys. Res. Commun. 458 (2015) 733-738.

[60] F.Z. Marques, C.R. Mackay, D.M. Kaye, Beyond gut feelings: how the gut microbiota regulates blood pressure, Nat. Rev. Cardiol. 15 (2018) 20-32.

[61] J.L. Pluznick, Microbial short-chain fatty acids and blood pressure regulation, Curr. Hypertens. Rep. 19 (2017) 25.

[62] A.J. Brown, S.M. Goldsworthy, A.A. Barnes, M.M. Eilert, L. Tcheang, D. Daniels, A.I. Muir, M.J. Wigglesworth, I. Kinghorn, N.J. Fraser, N.B. Pike, J.C. Strum, K.M. Steplewski, P.R. Murdock, J.C. Holder, F.H. Marshall, P.G. Szekeres, S. Wilson, D.M. Ignar, S.M. Foord, A. Wise, S.J. Dowell, The Orphan G protein-coupled receptors GPR41 and GPR43 are activated by propionate and other short chain car boxylic acids, J. Biol. Chem. 278 (2003) 11312-11319.

[63] J.L. Pluznick, R.J. Protzko, H. Gevorgyan, Z. Peterlin, A. Sipos, J. Han, I. Brunet, L.X. Wan, F. Rey, T. Wang, S.J. Firestein, M. Yanagisawa, J.I. Gordon, A. Eichmann, J. Peti-Peterdi, M.J. Caplan, Olfactory receptor responding to gut microbiota-derived signals plays a role in renin secretion and blood pressure regulation, Proc. Natl. Acad. Sci. U. S. A. 110 (2013) 4410-4415.

[64] J.L. Pluznick, Renal and cardiovascular sensory receptors and blood pressure regulation, Am. J. Physiol. Renal Physiol. 305 (2013) F439-F444.

[65] S.P. Whelton, A.D. Hyre, B. Pedersen, Y. Yi, P.K. Whelton, J. He, Effect of dietary fiber intake on blood pressure: a meta-analysis of randomized controlled clinical trials, J. Hypertens. 23 (2005) 475-481.

[66] E. Holmes, R.L. Loo, J. Stamler, M. Bictash, I.K. Yap, Q. Chan, T. Ebbels, I.M. De, I.J. Brown, K.A. Veselkov, M.L. Daviglus, H. Kesteloot, H. Ueshima, L. Zhao, J.K. Nicholson, P. Elliott, Human metabolic phenotype diversity and its association with diet and blood pressure, Nature 453 (2008) 396-400.

[67] S. Khalesi, J. Sun, N. Buys, R. Jayasinghe, Effect of probiotics on blood pressure: a systematic review and meta-analysis of randomized controlled trials, Hypertension 64 (2014) 897-903.

[68] M.D. Pagel, S. Ahmad, J.E. Vizzo, B.H. Scribner, Acetate and bicarbonate fluctuations and acetate intolerance during dialysis, Kidney Int. 21 (1982) 513-518.

[69] M.K. Raizada, B. Joe, N.S. Bryan, E.B. Chang, F.E. Dewhirst, G.G. Borisy, Z.S. Galis, W. Henderson, P.A. Jose, C.J. Ketchum, J.W. Lampe, C.J. Pepine, J.L. Pluznick, D. Raj, D.R. Seals, R.A. Gioscia-Ryan, W.H.W. Tang, Y.S. Oh, Report of the national heart, lung, and blood institute working group on the role of microbiota in blood pressure regulation: current status and future directions, Hypertension (2017) (ePUB ahead of publication).

[70] T. Yang, M.M. Santisteban, V. Rodriguez, E. Li, N. Ahmari, J.M. Carvajal, M. Zadeh, M. Gong, Y. Qi, J. Zubcevic, B. Sahay, C.J. Pepine, M.K. Raizada, M. Mohamadzadeh, Gut dysbiosis is linked to hypertension, Hypertension 65 (2015) 1331-1340.

[71] K.M. Maslowski, A.T. Vieira, A. Ng, J. Kranich, F. Sierro, D. Yu, H.C. Schilter, M.S. Rolph, F. Mackay, D. Artis, R.J. Xavier, M.M. Teixeira, C.R. Mackay, Regulation of inflammatory responses by gut microbiota and chemoattractant receptor GPR43, Nature 461 (2009) 1282-1286.

[72] C.W. McIntyre, L.E. Harrison, M.T. Eldehni, H.J. Jefferies, C.C. Szeto, S.G. John, M.K. Sigrist, J.O. Burton, D. Hothi, S. Korsheed, P.J. Owen, K.B. Lai, P.K. Li, Circulating endotoxemia: a novel factor in systemic inflammation and cardiovascular disease in chronic kidney disease, Clin. J. Am. Soc. Nephrol. 6 (2011) 133-141.

[73] E. Ritz, Intestinal-renal syndrome: mirage or reality? Blood Purif. 31 (2011) 70-76.

[74] P. Kotanko, M. Carter, N.W. Levin, Intestinal bacterial microflora-a potential source of chronic inflammation in patients with chronic kidney disease, Nephrol. Dial. Transplant. 21 (2006) 2057-2060.

[75] E. Metchnikoff, The Prolongation of Life - Optimistic Studies, (1908).

[76] N. Thevaranjan, A. Puchta, C. Schulz, A. Naidoo, J.C. Szamosi, C.P. Verschoor,
D. Loukov, L.P. Schenck, J. Jury, K.P. Foley, J.D. Schertzer, M.J. Larche, D.J. Davidson, E.F. Verdu, M.G. Surette, D.M.E. Bowdish, Age-associated microbial dysbiosis promotes intestinal permeability, systemic inflammation, and macrophage dysfunction, Cell Host Microbe 21 (2017) 455-466.

[77] M. Bossola, M. Sanguinetti, D. Scribano, C. Zuppi, S. Giungi, G. Luciani, R. Torelli, B. Posteraro, G. Fadda, L. Tazza, Circulating bacterial-derived DNA fragments and markers of inflammation in chronic hemodialysis patients, Clin. J. Am. Soc. Nephrol. 4 (2009) 379-385.

[78] J.J. Carrero, P. Stenvinkel, Inflammation in end-stage renal disease-what have we learned in 10 years? Semin. Dial. 23 (2010) 498-509.

[79] D.S. Raj, J.J. Carrero, V.O. Shah, A.R. Qureshi, P. Barany, O. Heimburger, B. Lindholm, J. Ferguson, P.L. Moseley, P. Stenvinkel, Soluble CD14 levels, interleukin 6, and mortality among prevalent hemodialysis patients, Am. J. Kidney Dis. 54 (2009) 1072-1080.

[80] D.S. Raj, V.O. Shah, M. Rambod, C.P. Kovesdy, K. Kalantar-Zadeh, Association of soluble endotoxin receptor CD14 and mortality among patients undergoing hemodialysis, Am. J. Kidney Dis. 54 (2009) 1062-1071.

[81] R. Poesen, A. Ramezani, K. Claes, P. Augustijns, D. Kuypers, I.R. Barrows, J. Muralidharan, P. Evenepoel, B. Meijers, D.S. Raj, Associations of soluble CD14 and endotoxin with mortality, cardiovascular disease, and progression of kidney disease among patients with CKD, Clin. J. Am. Soc. Nephrol. 10 (2015) 1525-1533.

[82] R. Ross, Atherosclerosis-an inflammatory disease, N. Engl. J. Med. 340 (1999) $115-126$

[83] P.M. Ridker, B.M. Everett, T. Thuren, J.G. MacFadyen, W.H. Chang, C. Ballantyne, F. Fonseca, J. Nicolau, W. Koenig, S.D. Anker, J.J.P. Kastelein, J.H. Cornel, P. Pais, D. Pella, J. Genest, R. Cifkova, A. Lorenzatti, T. Forster, Z. Kobalava, L. Vida-Simiti, M. Flather, H. Shimokawa, H. Ogawa, M. Dellborg, P.R.F. Rossi, R.P.T. Troquay, P. Libby, R.J. Glynn, Antiinflammatory therapy with canakinumab for atherosclerotic disease, N. Engl. J. Med. 377 (2017) 1119-1131.

[84] S. Lekawanvijit, A.R. Kompa, H. Krum, Protein-bound uremic toxins: a long overlooked culprit in cardiorenal syndrome, Am. J. Physiol. Renal Physiol. 311 (2016) F52-F62.

[85] R. Poesen, B. Meijers, P. Evenepoel, The colon: an overlooked site for therapeutics in dialysis patients, Semin. Dial. 26 (2013) 323-332.

[86] A. Ramezani, D.S. Raj, The gut microbiome, kidney disease, and targeted interventions, J. Am. Soc. Nephrol. 25 (2014) 657-670.

[87] N.D. Vaziri, J. Yuan, K. Norris, Role of urea in intestinal barrier dysfunction and disruption of epithelial tight junction in chronic kidney disease, Am. J. Nephrol. 37 (2013) 1-6.

[88] G. Schulman, T. Berl, G.J. Beck, G. Remuzzi, E. Ritz, K. Arita, A. Kato, M. Shimizu, Randomized placebo-controlled EPPIC trials of AST-120 in CKD, J. Am. Soc. Nephrol. 26 (2015) 1732-1746.

[89] V.M. Brandenburg, G. Schlieper, N. Heussen, S. Holzmann, B. Busch, P. Evenepoel, R. Vanholder, B. Meijers, N. Meert, W.J. Fassbender, J. Floege, W. Jahnen-Dechent, M. Ketteler, Serological cardiovascular and mortality risk predictors in dialysis patients receiving sevelamer: a prospective study, Nephrol. Dial. Transplant. 25 (2010) 2672-2679.

[90] B. Guida, M. Cataldi, E. Riccio, L. Grumetto, A. Pota, S. Borrelli, A. Memoli, F. Barbato, G. Argentino, G. Salerno, B. Memoli, Plasma p-cresol lowering effect of sevelamer in peritoneal dialysis patients: evidence from a Cross-Sectional Observational Study, PLoS One 8 (2018) 2013-e73558.

[91] R. Masereeuw, J. Löwenstein, T. Toyohara, A. Sparreboom, D. Sweet, R. Mutsaers, The kidney and uremic toxin removal: glomerulus or tubulus? Semin. Nephrol. (2016) 2014

[92] T.W. Meyer, E.C. Leeper, D.W. Bartlett, T.A. Depner, Y.Z. Lit, C.R. Robertson, T.H. Hostetter, Increasing dialysate flow and dialyzer mass transfer area coefficient to increase the clearance of protein-bound solutes, J. Am. Soc. Nephrol. 15 (2004) 1927-1935.

[93] B.K.I. Meijers, B. Bammens, K. Verbeke, P. Evenepoel, A review of albumin binding in CKD, Am. J. Kidney Dis. 51 (2008) 839-850.

[94] T.W. Meyer, J.W. Peattie, J.D. Miller, D.C. Dinh, N.S. Recht, J.L. Walther, T.H. Hostetter, Increasing the clearance of protein-bound solutes by addition of a sorbent to the dialysate, J. Am. Soc. Nephrol. 18 (2007) 868-874.

[95] B.K.I. Meijers, V. Weber, B. Bammens, W. Dehaen, K. Verbeke, D. Falkenhagen, P. Evenepoel, Removal of the uremic retention solute p-cresol using fractionated plasma separation and adsorption, Artif. Org. 32 (2008) 214-219. 